\title{
A Posteriori Error Estimate for Streamline Diffusion Method in Solving a Hyperbolic Equation
}

\author{
Davood Rostamy, Fatemeh Zabihi \\ Department of Mathematics, Imam Khomeini International University, Qazvin, Iran \\ E-mail: rostamy@khayam.ut.ac.ir \\ Received December 23, 2010; revised May 30, 2011; accepted June 7, 2011
}

\begin{abstract}
In this article, we use streamline diffusion method for the linear second order hyperbolic initial-boundary value problem. More specifically, we prove a posteriori error estimates for this method for the linear wave equation. We observe that this error estimates make finite element method increasingly powerful rather than other methods.
\end{abstract}

Keywords: Streamline Diffusion Method, Hyperbolic Problems, Wave Equations, Finite Element, A Posteriori Error Estimate

\section{Introduction}

The wave equation based on rigorous a posteriori error estimates is a largely subject, despite the importance of these problems in the modeling of a number of physical phenomena. A posteriori have made every method increasingly powerful; such that there are various approaches to a posteriori error estimates and it has new successfully applied to varied problems by several authors (see Ainsworth and Tinsley Oden [1]; Asadzadeh [2]; Gergouli [3]; Johnson [4] and [5]).

Gergouli et al. [3] and his teammates applied finite element method for linear wave equation and obtained a posteriori error estimates in $L\left(L^{2}\right)$ norm in Johnson proved existence solution for second order hyperbolic problems and used discontinuous Galerkin method for them and obtained a priori and a posteriori error estimates. In this paper, we do new work and use streamline diffusion method (SD-method) for solving the linear second order hyperbolic initial-boundary value problem.

Streamline diffusion methods (Asadzadeh [6]; Asadzadeh and Kowalczyk [7]; Eriksson and Johnson [8]; Brenner [9]; Dubois [10]; Fuhrer [11] ) perform slightly better than the standard finite element methods for smooth solutions and non-smooth solutions hyperbolic problems as a two-dimensional one which both is higher order accurate and has good stability properties. Due to the fact that artificial diffusion is added only in the characteristic direction so that internal layers are not smeared out, while the added diffusion removes oscillations near boundary layers.

We consider the linear second order hyperbolic initial boundary value problem (see Codina [12]; Haws, [13]; Gergoulus et al., [3]; Iraniparast [14]; Kalmenov [15]; in Sobolov space Adams [16]; Shermenew [17]) as follows:

$$
\begin{cases}u_{t t}-\nabla \cdot(a \nabla u)=f & \text { in } \Omega \times(0, T) \\ u\left(x, 0=u_{0}(x)\right) & \text { on } \Omega \times\{0\} \\ u_{t}(x, 0)=u_{1}(x, 0) & \text { on } \Omega \times\{0\} \\ u(x, 0)=0 & \text { on } \partial \Omega \times(0, T]\end{cases}
$$

Here, $\Omega \subset \Re^{d}$ is a bounded open polygonal domain with boundary $\partial \Omega$ and we have $u_{0} \in H_{0}^{1}(\Omega), u_{1} \in L^{2}(\Omega)$, a is a scalar-value function in $C(\bar{\Omega})$ and $f \in L^{2}\left(0, T ; L^{2}(\Omega)\right)$.

For (1), we use one variable changing and obtain a new problem. We apply SD-method for new problem and obtain a posteriori error estimates. A posteriori error bound provides a computable upper bound on the error in some norm using the computed finite element solution (see Ainsworth and Tinsley Oden [1]; Asadzadeh [2]; Burman [18]; Johnson and Szepessy [19]; Sandboge [20]).

In order to make use of the theory of Semigroups we write the system (1) in the following abstract form: 


$$
\begin{cases}w_{t}+A w=F & \text { in }(\Omega \times(0, T))^{2} \\ w(x, 0)=w_{0}(x) & \text { on }(\Omega \times\{0\})^{2} \\ w(0, t)=0 & \text { on }(\partial \Omega \times(0, T])^{2}\end{cases}
$$

Here, we assume $v=u_{t}$ for $x \in \mathbb{R}^{d}$ and $t \in[0, T]$, also:

$$
\begin{aligned}
w(x, t) & =(u(x, t), v(x, t))^{\mathrm{T}}, w_{t}(x, t)=\left(u_{t}(x, t), v_{t}(x, t)^{\mathrm{T}}\right) \\
\boldsymbol{A} & =\left(\begin{array}{cc}
0 & -\boldsymbol{I} \\
\nabla \cdot(a \nabla) & 0
\end{array}\right) w_{0}(x)=\left(u_{0}(x), u_{1}(x)\right)^{\mathrm{T}}
\end{aligned}
$$

and

$$
F(x, t)=(0, f(x, t))^{\mathrm{T}}
$$

where, $\boldsymbol{I}$ is identity matrix.

The rest of this study is organized as follows. In Section 2, we define slabs for space-time domain and obtain SD-method for (2) this slabs. In Section 3, we consider a posteriori error estimates for SD-method form of Section 2 and obtain dual problem. In Section 4, we define interpolation estimates for dual problem. In Section 5, we complete proof for a posteriori error estimates by using definitions in Section 4.

\section{The Streamline Diffusion Method}

In this section, we consider the SD-method for solving (2) that is based on using finite element over the space-time domain $\Omega \times[0, T]$. To define this method, let $0=t_{0}<t_{1}<\cdots<t_{N}=T$ be a subdivision of the time interval $[0, T]$ into intervals $I_{n}=\left(t_{n}, t_{n+1}\right)$, with time steps $k_{n}=t_{n+1}-t_{n}, n=0,1, \cdots, N-1$ and introduce the corresponding space-time slabs, i.e.:

$$
S_{n=}\left\{(x, t): x \in, t_{n}<t<t_{n+1}\right\}
$$

for $n=0,1, \cdots, N-1$. Further, for each n let $W^{n}$ be a finite element subspace of $H^{1}\left(S_{n}\right) \times H^{1}\left(S_{n}\right)$, (see Adams, [16]) and let:

$$
\dot{W}^{n}=\left\{w \in W^{n} \mid w(0, t)=0, \text { for } t \in I_{n}\right\}
$$

We can formulate SD-method on the slab $S_{n}$ for (2), as follows:

For $n=0, \cdots, N-1$, find $w^{n} \in \dot{W}$ such that:

$$
\begin{aligned}
& \left(w_{t}^{n}+A w^{n}, g+\delta\left(g_{t}+A g\right)\right)_{n}+<w_{+}^{n}, g+>_{n} \\
& =\left(F, g+\delta\left(g_{t}+A g\right)\right)_{n}+<w_{-}^{n}, g_{+}>_{n}
\end{aligned}
$$

where, $\delta=\bar{C} h$ with $\bar{C}$ is a suitable chosen (sufficiently small, see Johnson, [18]) positive constant and parameter $h$ is defined in the following. Further, we de- fine the following notations for (6):

$$
\begin{gathered}
(w, g)_{n}=\int_{S n} w^{T} \cdot g \mathrm{~d} x \mathrm{~d} t \\
<w, g>_{n}=\int_{\Omega} w^{T}\left(x, t_{n}\right) \cdot g\left(x, t_{n}\right) \mathrm{d} x \\
w+(x, t)=\lim _{s \rightarrow 0+} w(x, t+s) \lim _{s \rightarrow 0+} \\
w-(x, t)=\lim _{s \rightarrow 0-} w(x, t+s)
\end{gathered}
$$

The terms including $<,>$ in the above formula is a jump conditions which imposes a weakly enforced continuity condition across the slab interfaces, at $t_{n}$ and is the mechanism by which information is propagated from one slab to another. For more concisely, after summing over $n$, we may rewrite (5) as follow:

We assume $\dot{w}=\coprod_{n=0}^{N-1} \dot{W}^{n}$ and find $\mid w \in \dot{w}$, such that:

$$
B(w, g)=L(g)
$$

For $g \in \dot{w}$ and where the bilinear form $B(.,$.$) and$ the linear form $L($.$) define by:$

$$
\begin{aligned}
B(w, g) & =\sum_{n=0}^{N-1}\left(w_{t}^{n}+A w^{n}, g+\delta\left(g_{t}+A g\right)\right)_{n} \\
& +\sum_{n=1}^{N-1}<\left[w^{n}\right], g_{+}>_{n}+<w_{+}^{n} g_{+}>_{0}
\end{aligned}
$$

where, we define $w=\left(w_{1}, w_{2}\right)^{\mathrm{T}}$ such that for $i=1,2$ :

$$
\left[w_{i}\right]=w_{i,+}-w_{i,-}, \quad[w]=\left(\left[w_{1}\right],\left[w_{2}\right]\right)^{\mathrm{T}}
$$

and

$$
L(g)=\sum_{n=0}^{N-1}(F, g+\delta(g t+A g))_{n}+<w_{0}, g+>_{0}
$$

For $h>0$,we define $T_{h}^{n}$ such that be a triangulation of the slab $S_{n}$ into triangles $K$ satisfying as usual the minimum angle condition (Ciarlet [21]) and assume that the parameter $h$ is represented with the maximum diameter of the triangles $K \in T_{h}^{n}$. We introduce:

$$
\begin{gathered}
W_{h}^{n}=\left\{w \in H^{1}\left(S_{n}\right) \times H^{1}\left(S_{n}\right):\left.w\right|_{k} \in P_{k}(K) \times P_{k}(K)\right. \\
\text { for } \left.\in T_{h}^{n}, w(0, t)=0 \text { for } t \in I_{n}\right\}
\end{gathered}
$$

where, $P_{k}(K)$ denotes the set of polynomials in $K$ of degree less than or equal $\mathrm{k}$ and:

$$
W_{h}=\prod_{n=0}^{N-1} W_{h}^{n}
$$

Thus (6) can be formulated as follows:

Find $w_{h} \in W_{h}$ such that:

$$
B\left(w_{h}, g\right)=L(g)
$$

for $g \in W_{h}$. Moreover, we know that the exact solution of (6) satisfies: 


$$
B(w, g)=L(g)
$$

for $g \in \dot{w}$ and by use (6) and (7), we have the Galerkin orthogonality relation:

$$
B(e, g)=0
$$

where, $e=w-w_{h}$.

\section{An a Posteriori Error Estimate for the SD-Method}

In this section, we shall consider the following simplified version of SD-method for (7) with $\delta=0$ : Find $w_{h} \in W_{h}$, such that for $n=0,1, \cdots, N-1$ :

$$
\begin{aligned}
& \sum_{n=0}^{N-1}\left(w_{h, t}^{n}+A w_{h}^{n}, g\right)_{n}+\sum_{n=1}^{N-1}<\left[w_{h}^{n}\right], g+>_{n}+<w_{h,+}^{n}, g+>_{0} \\
& =\sum_{n=0}^{N-1}(F, g)_{n}+<w_{0}, g+>_{0}
\end{aligned}
$$

where, $g \in W_{h}$ and $w_{h,-}^{0}=0$.

For simplicity, we take $w_{0}=0$ and $F=0$. In order to obtain a representation of the error, we consider the following auxiliary problem, referred to as the linearized dual problem: Find $\Phi$ such that:

$$
\left\{\begin{array}{l}
L^{*} \Phi \equiv-\Phi_{t}+A^{T} \Phi=\Psi^{-1} e \text { in } \Omega \\
\Phi(0, t)=0, t \in(0, T) \\
\Omega(x, T)=0, x \in \Omega
\end{array}\right.
$$

and $L^{*}$ denotes the adjoint of the operator $L$ defined in (2) and $\psi$ is a positive weight function. Note that this problem is computed "backward", but there is a corresponding change in sign. Further, we shall first introduce the following notation:

$$
\|w\|_{L_{2}^{\psi}(\Omega)}=(w, \Psi w)_{\Omega}^{1 / 2}
$$

Multiplying (10) by $e$ and integrating by parts and summing over $n$, we obtain the following error representation formula:

$$
\begin{aligned}
\|e\|_{L_{2}^{\psi-1}(\Omega)} & =\left(e, \Psi^{-1} e\right)_{\Omega}=\left(e, L^{*} \Phi\right)_{\Omega} \\
& =\sum_{n=0}^{N-1}\left(e,-\Phi_{t}+A^{T} \Phi\right)_{n} \\
& =\sum_{n=0}^{N-1}\left(e,-\Phi_{t}\right)_{n}+\sum_{n=0}^{N-1}\left(e, A^{T} \Phi\right)_{n}
\end{aligned}
$$

We have for $n=0,1, \cdots, N-1$ by part integrating:

$$
\begin{aligned}
\left(e, \Phi_{t}\right)_{n} & =-\int_{S_{n}} e^{T} \cdot \Phi_{t} \mathrm{~d} x \mathrm{~d} t \\
& =-\sum_{n=0}^{N-1} \int_{\Omega} e^{T}\left(x, t_{n}\right) \cdot \Phi\left(x, t_{n}\right) \mathrm{d} x+\int_{S_{n}} e_{t}^{T} \cdot \Phi \mathrm{d} x \mathrm{~d} t \\
& =-\sum_{n=0}^{N-1} \int_{\Omega} e^{T}\left(x, t_{n}\right) \cdot \Phi\left(x, t_{n}\right) \mathrm{d} x+\left(e_{t}, \Phi\right)_{n}
\end{aligned}
$$

We define $e=\left(e_{1}, e_{2}\right)^{\mathrm{T}}$ and $\Phi=\left(\phi_{1}, \phi_{2}\right)^{\mathrm{T}}$ and obtain for $n=0,1, \cdots, N-1$ :

$$
\begin{aligned}
\left(e, A^{T} \Phi\right)_{n} & =\int_{S_{n}} e^{T} \cdot A^{T} \Phi \mathrm{d} x \mathrm{~d} t \\
& =\int_{S_{n}} e^{T} \cdot\left(\begin{array}{cc}
0 & -\nabla \cdot(a \nabla) \\
-1 & 0
\end{array}\right)\left(\begin{array}{l}
\phi_{1} \\
\phi_{2}
\end{array}\right) \mathrm{d} x \mathrm{~d} t \\
& =\int_{S_{n}}\left(e_{1} e_{2}\right) \cdot\left(\begin{array}{c}
-\nabla \cdot\left(a \nabla \phi_{2}\right) \\
-\phi_{1}
\end{array}\right) \mathrm{d} x \mathrm{~d} t \\
& =\int_{S_{n}}\left(-e_{1} \nabla \cdot\left(a \nabla \phi_{2}\right)-e_{2} \phi_{1}\right) \mathrm{d} x \mathrm{~d} t \\
& =\int_{S_{n}}\left(a \nabla \phi_{2} \nabla e_{1}-e_{2} \phi_{1}\right) \mathrm{d} x \mathrm{~d} t \\
& =\int_{S_{n}}\left(-\nabla \cdot\left(a \nabla \phi_{2}\right) e_{1}-e_{2} \phi_{1}\right) \mathrm{d} x \mathrm{~d} t \\
& =\int_{S_{n}}(A e)^{T} \cdot \Phi \mathrm{d} x \mathrm{~d} t=(A e, \Phi)_{n}
\end{aligned}
$$

We define:

$$
\begin{aligned}
J & =\sum_{n=0}^{N-1} \int_{\Omega} e^{T}\left(x, t_{n}\right) \cdot \Phi\left(x, t_{n}\right) d x \\
& =\left(<e_{-}, \Phi_{-}>_{1}-<e_{+}, \Phi_{+}>_{0}\right) \\
& +\left(<e_{-}, \Phi_{-}>_{2}-<e_{+}, \Phi_{+}>_{1}\right) \\
& +\cdots+\left(<e_{-}, \Phi_{-}>_{N-1}-<e_{+}, \Phi_{+}>_{N-2}\right) \\
& +\left(<e_{-}, \Phi_{-}>_{N}-<e_{+}, \Phi_{+}>_{N-1}\right) \\
& =-\left[<e_{-}, \Phi_{-}>_{N}+<e_{+}, \Phi_{+}>_{0}\right. \\
& \left.+\sum_{n=0}^{N-1}<[e], \Phi_{+}>_{n}+\sum_{n=0}^{N-1}<e_{-},[\Phi]>_{n}\right]
\end{aligned}
$$

According to (9), $\phi\left(., t_{N}=T\right)=0$ and since $e_{-}^{0}=\left[w_{0}\right]=0$, we get:

$$
J=\sum_{n=0}^{N-1}<\left[w_{h}\right], \Phi_{+}>_{n}
$$

Then in (12), by use (15) and (16), we have:

$$
\|e\|_{L_{2}^{\psi+1}(\Omega)}^{2}=\sum_{n=0}^{N=1}\left(e_{t}, \Phi\right)+\sum_{n=0}^{N=1}(A e, \Phi)_{n}+\sum_{n=0}^{N=1}<\left[w_{h}\right], \Phi_{+}>_{n}
$$

So that recalling (9) and using the Galerkin orthogonality (8), we obtain: 


$$
\begin{aligned}
\|e\|_{L_{2}^{\psi+1}(\Omega)}^{2} & =\sum_{n=0}^{N=1}\left(e_{t}, \Phi\right)+\sum_{n=0}^{N=1}(A e, \Phi)_{n}+\sum_{n=0}^{N=1}<\left[w_{h}\right], \Phi_{+}>_{n} \\
& =\sum_{n=0}^{N-1}\left(\left(w-w_{h}\right)_{t}+A\left(w-w_{h}\right), \Phi\right)_{n} \\
& +\sum_{n=0}^{N-1}<\left[w_{h}\right], \Phi_{+}>_{n}=\sum_{n=0}^{N-1}\left(F-w_{h, t}-A w_{h}, \Phi\right)_{n} \\
& +\sum_{n=0}^{N-1}<\left[w_{h}\right], \Phi_{+}>_{n}
\end{aligned}
$$

where, $\hat{\Phi} \in W_{h}$ is an interpolant of $\Phi$. The idea is now to estimate $\hat{\Phi}-\Phi$ in terms of $\psi^{-1} e$ using a strong stability estimates for solution $\Phi$ of the dual problem.

\section{Interpolation Estimates for the Dual Solution}

We shall now consider our interpolant $\hat{\Phi} \in W_{h}$ in (16) to be the space-time $L_{2}$-projection of $\Phi$, namely if the first, we define the $L_{2}$-projections:

$$
P_{n}: L_{2}(\Omega) \mapsto W_{h}^{n}
$$

and

$$
\begin{aligned}
& \pi_{n}: L_{2}\left(S_{n}\right) \mapsto \prod_{0, n}=\left\{w \in L_{2}\left(S_{n}\right): w(x, .)\right. \\
& \text { is constant on } \left.I_{n}, \quad x \in \Omega\right\}
\end{aligned}
$$

in space and in space time, respectively, by:

$$
\begin{gathered}
\int_{\Omega}\left(P_{n}, \Phi\right)^{T} \cdot w \mathrm{~d} x=\int_{\Phi} \Phi^{T} \cdot w \mathrm{~d} x, \quad \forall w \in W_{h}^{n} \\
\pi_{n} w \mid S_{n}=\frac{1}{k_{n}} \int_{I_{n}} w(., t) \mathrm{d} t, \quad \forall w \in \prod_{0, n}
\end{gathered}
$$

Then, we can define $\hat{\Phi} \mid S_{n} \in W_{h}^{n}$ by letting:

$$
\hat{\Phi} \mid S_{n}=P_{n} \pi_{n} \Phi=\pi_{n} P_{n} \Phi \in W_{h}^{n}
$$

where, $\Phi=\Phi \mid S_{n}$. Further, if we introduce $P$ and $\pi$ defined by:

$$
(P \Phi) \mid S_{n}=P_{n}\left(\Phi \mid S_{n}\right)
$$

and

$$
(\pi \Phi) \mid S_{n}=\pi_{n}\left(\Phi \mid S_{n}\right)
$$

respectively, then we can let $\hat{\Phi} \in W_{h}$ to be:

$$
\hat{\Phi}=P \pi \Phi=\pi P \Phi \in W_{h}
$$

Now, we define residual of computed solution $w_{h}$ by:

$$
\begin{gathered}
R_{0}=F-w_{\mathrm{h}, \mathrm{t}}-A w_{\mathrm{h}} \\
R_{1}=w_{h,+}^{n}-w_{h,-}^{n} \text {, on } S_{n}
\end{gathered}
$$

$$
R_{2}=\frac{\left(P_{n}-I\right) w_{h,-}^{n}}{k_{n}} \text {, on } S_{n}
$$

where, $I$ is the identity operator.

In the end of this section, we shall give a lemma for interpolation estimates by the projection operators $P$, leaving the overall of $I$ and $I I$ to next section.

Lemma 1: There is a constant $C$ such that for residual $R \in L_{2}(\Omega)$ :

$$
\left|(R, \Phi-P \Phi)_{\Omega}\right| \leq C\left\|h^{2}(I-P) R\right\|_{L_{2}^{\Psi-1}(\Omega)}\left\|\Phi_{x x}\right\|_{L_{2}^{\Psi}(\Omega)}
$$

Proof: (see Johnson and Szepessy [19] and Sandboge [20]).

\section{The Completion of the Proof of a Posteriori Error Estimates}

In this section we state and prove a posteriori error estimate by estimating of the terms $I$ and $I I$ in the error representation formula (16). To this approach we introduce the stability factors (see Burman [18]) associated with discretization in time and space, defined by:

$$
\gamma_{e}^{t}=\frac{\left\|\Phi_{t}\right\|_{L_{2}^{\psi}(\Omega)}}{\|e\|_{L_{2}^{\psi-1}(\Omega)}}
$$

and

$$
\gamma_{e}^{x}=\frac{\left\|\Phi_{x x}\right\|_{L_{2}^{\Psi}(\Omega)}}{\|e\|_{L_{2}^{\Psi-1}(\Omega)}}
$$

respectively. We now apply the result of the previous sections; using Catchy-Schwartz inequality in (16) coupled with the interpolation estimate (17) and the strong stability factors (18) and (19), to derive the $L^{2}\left(L^{2}\right)$ a posteriori error estimates for the scheme (9).

Theorem 1: The error $e=w-w_{h}$, where $w$ is the solution of the continuous problems (2) and $w_{h}$ that of (9), satisfies the following stability estimate:

$$
\begin{aligned}
\|e\|_{L_{2}^{\psi-1}(\Omega)} & \leq C \gamma_{e}^{x}\left\|h^{2}(I-P) R_{0}\right\|_{L_{2}^{\psi-1}(\Omega)}+C \gamma_{e}^{x}\left\|k_{n} R_{1}\right\|_{L_{2}^{\psi-1}(\Omega)} \\
& +\gamma_{e}^{x}\left\|h^{2} R_{2}\right\|_{L_{2}^{\psi-1}(\Omega)}+\gamma_{e}^{x}\left\|k_{n} R_{2}\right\|_{L_{2}^{\psi-1}(\Omega)}
\end{aligned}
$$

Proof: Using the notation introduce above, we may write (17) as:

$$
\begin{aligned}
\|e\|_{L_{2}^{\Psi-1}(\Omega)}^{2} & =\sum_{n=0}^{N-1}\left(R_{0}, \hat{\Phi}-\Phi\right)_{n}+\sum_{n=0}^{N-1}<k_{n} \frac{\left[w_{h}\right]}{k_{n}},(\hat{\Phi}-\Phi)_{+}>_{n} \\
& =I+I I
\end{aligned}
$$

Below we shall estimate the terms $I$ and II separately. 
Splitting the interpolation error by writing

$\hat{\Phi}-\Phi=\hat{\Phi}-P \Phi+P \Phi-\Phi$ and $\hat{\Phi}_{n}=\pi_{n} P \Phi$, we have:

$$
\begin{aligned}
I= & \sum_{n=0}^{N-1}\left(R_{0} \hat{\Phi}-P \Phi+P \Phi-\Phi\right)_{n} \\
= & \sum_{n=0}^{N-1}\left(R_{0}, \hat{\Phi}_{n}-P \Phi\right)_{n}+\sum_{n=0}^{N-1}\left(R_{0}, P \Phi-\Phi\right)_{n} \\
& \leq C\left\|h^{2}(I-P) R_{0}\right\|_{L_{2}^{\Psi-1}(\Omega)}\left\|\Phi_{x x}\right\|_{L_{2}^{\Psi}(\Omega)}
\end{aligned}
$$

where we have used the fact that $R_{0}$ is constant in the time, (making the first integral zero) and then using interpolation estimate (17) in the second integral. It remains to estimate the terms $I I$, to this end, we need the following notation:

$$
\Phi_{+}^{n}(x)=\Phi(x, t)-\int_{t_{n}}^{t} \frac{\partial}{\partial \tau} \Phi(x, \tau) \mathrm{d} \tau \mathrm{d} t
$$

so that:

$$
k_{n} \Phi_{+}^{n}(x)=\int_{I_{n}} \Phi(x, t) \mathrm{d} t-\int_{I_{n}} \int_{I_{n}}^{t} \Phi_{\tau}(x, \tau) \mathrm{d} \tau \mathrm{d} t
$$

where, $\Phi_{\tau}=\frac{\partial \Phi}{\partial \tau}$ and $\hat{\Phi}_{n}=\hat{\Phi}\left(., t_{n}\right)$ :

$$
\begin{aligned}
I I & =\sum_{n=0}^{N-1}\left\langle k_{n} \mid \frac{\left[w_{h}\right]}{k_{n}},(\hat{\Phi}-\Phi)_{+}\right\rangle_{n} \\
& =\sum_{n=0}^{N-1}\left\langle k_{n} \frac{\left[w_{h}\right]}{k_{n}},\left(\hat{\Phi}_{n}-P \Phi+P \Phi-\Phi\right)_{+}\right\rangle_{n} \\
& =\sum_{n=0}^{N-1}\left\langle k_{n} \frac{w_{h}}{k_{n}},\left(\hat{\Phi}_{n}-P \Phi\right)+\right\rangle_{n} \\
& +\sum_{n=0}^{N-1}\left\langle k_{n} \frac{\left[w_{h}\right]}{k_{n}},(P \Phi-\Phi)_{+}\right\rangle_{n}: I I_{1}+I I_{2}
\end{aligned}
$$

To estimate $I I_{1}$, we use (21) to get:

$$
\begin{aligned}
I I_{1} & =\sum_{n=0}^{N-1}\left\langle k_{n} R_{1},\left(\hat{\Phi}_{n}\right)_{+}-P \Phi_{+}\right\rangle_{n} \\
= & \sum_{n=0}^{N-1}\left\langle R_{1} k_{n} \hat{\Phi}_{n}-P k_{n} \Phi_{+}\right\rangle_{n} \\
= & \sum_{n=0}^{N-1}\left\langle R_{1}, k_{n} \hat{\Phi}_{n}-\int_{I_{n}} P \Phi(., t) \mathrm{d} t+\int_{I_{n}} \int_{t_{n}}^{t} P \Phi_{\tau}(., \tau) \mathrm{d} \tau \mathrm{d} t\right\rangle_{n} \\
& =\sum_{n=0}^{N-1} \int_{I_{n}} \int_{t_{n}}^{t}\left\langle R_{1} P \Phi_{\tau}(., \tau)\right\rangle_{n} \mathrm{~d} \tau \mathrm{d} t \\
& \leq\left\|k_{n} R_{2}\right\|_{L_{2}^{\Psi-1}(\Omega)}\left\|P \Phi_{t}\right\|_{L_{2}^{\Psi}(\Omega)} \\
& \leq\left\|k_{n} R_{1}\right\|_{L_{2}^{\Psi-1}(\Omega)}\left\|\Phi_{t}\right\|_{L_{2}^{\Psi}(\Omega)}
\end{aligned}
$$

As for the $I I_{2}$-terms we can write:

$$
\begin{aligned}
I I_{2}= & \sum_{n=0}^{N-1}\left\langle k_{n} \frac{\left[w_{h}\right]}{k_{n}}(P \Phi-\Phi)_{+}\right\rangle_{n} \\
& \cdot \sum_{n=0}^{N-1}\left\langle\frac{w_{h,+}^{n}-w_{h,-}^{n}}{k_{n}},\left(P_{n}-I\right) k_{n} \Phi_{+}\right\rangle_{n} \\
= & \sum_{n=0}^{N-1}\left\langle\frac{P_{n} w_{h,-}^{n}-w_{h,-}^{n}}{k_{n}},\left(P_{n}-I\right)\left(\int_{I_{n}} \Phi(., t) \mathrm{d} t\right.\right. \\
& \left.-\int_{I_{n}} \int_{t_{n}}^{t} \Phi_{\tau}(., \tau) \mathrm{d} \tau \mathrm{d} t\right\rangle_{n} \\
& \leq \sum_{n=0}^{N-1} \int_{I_{n}}\left\langle\frac{\left(P_{n}-I\right) w_{h,-}^{n}}{k_{n}},\left(P_{n}-I\right) \Phi(., t)\right\rangle_{n} \mathrm{~d} t \\
& +\sum_{n=0}^{N-1} \int_{I_{n}} \int_{I_{n}}\left\langle\frac{\left(P_{n}-I\right) w_{h,-}^{n}}{k_{n}},\left(P_{n}-I\right) \Phi_{\tau}(., t) \mathrm{d} \tau \mathrm{d} t\right\rangle_{n} \\
& \leq\left\|k_{n} R_{2}\right\|_{L_{2}^{\Psi-1}(\Omega)}\left\|\Phi_{x x}\right\|_{L_{2}^{\Psi}(\Omega)}+\left\|k_{n} R_{2}\right\|_{L_{2}^{\Psi-1}(\Omega)}\left\|\Phi_{t}\right\|_{L_{2}^{\Psi}(\Omega)}
\end{aligned}
$$

The a posteriori error estimate now follows immediately after collecting the terms and using the definition of the stability factors (18) and (19).

For $\delta \neq 0$ in (7), we can obtain a posteriori error estimates with similar way.

\section{References}

[1] M. Ainsworth and J. Tinsley Oden, "A Posteriori Error Estimation in Finite Element Analysis," Wiley-Interscience, New York, 2000.

[2] M. Asadzadeh, "A Posteriori Error Estimates for the Fokker-Planck and Fermi Pencil Beam Equations," Mathematical Methods in the Applied Sciences, Vol. 10, No. 3, 2000, pp. 737-769. doi:10.1142/S0218202500000380

[3] E. H. Gergouli, O. Lakkis and C. Makridakis, "A Posteriori $\mathrm{L}_{1}\left(\mathrm{~L}_{2}\right)$-Error Bounds in Finite Element Approximation of the Wave Equation," arXiv:1003.3641v1[math. NA], 2010, pp. 1-17.

[4] C. Johnson, "Numerical Solutions of Partial Differential Equations by the Finite Element Method," Cambridge University, Cambridge, 1987.

[5] C. Johnson, "Discontinous Galerkin Finite Element Methods for Second Order Hyperbolic Problems," Computer Methods in Applied Mechanics and Engineering, Vol. 107, No. 5, 1993, pp. 117-129. doi:10.1016/0045-7825(93)90170-3

[6] M. Asadzadeh, "Streamline Diffusion Methods for the Vlasov-Poisson Equations. RAIRO Math," Modelling and Numerical Analysis, Vol. 24, No. 3, 1990, pp. 177196.

[7] M. Asadzadeh and P. Kowalczyk, "Convergence of 
Streamline Diffusion Methods for the Vlasov-PoissonFokker-Planck System," Numerical Methods for Partial Differential Equations, Vol. 21, No. 2, 2005, pp. 472-495. doi:10.1002/num.20044

[8] K. Eriksson and C. Johnson, "Adaptive Streamline Diffusion Finite Element Methods for Stationary ConvectionDiffusion Problems," Mathematics of Computation, Vol. 60, No. 4, 1993, pp. 167-188. doi:10.1090/S0025-5718-1993-1149289-9

[9] S. C. Brenner and L. R. Scott, "The Mathematical Theory of Finite Element Method," Springer-Verlag, New York, 1994.

[10] F. Dubois and P. G. Le Floch, "Boundary Conditions for Nonlinear Hyperbolic Systems of Conservation Laws," Journal of Differential Equations, Vol. 71, No. 3, 2001, pp. 93-122.

[11] C. Fuhrer and R. Rannacher, "An Adaptive Streamline Diffusion Finite Element Method for Hyperbolic Conservation Laws," East-West Journal of Numerical Mathematics, Vol. 5, No. 2, 1997, pp. 145-162.

[12] R. Codina," Finite Element Approximation of the Hyperbolic Wave Equation in Mixed Form," Computer Methods in Applied Mechanics and Engineering, Vol. 197, No. 6, 2008, pp. 1305-1322. doi:10.1016/j.cma.2007.11.006

[13] L. Haws, "Symmetric Greens Functions for Certain Hyperbolic Problems," Computers \& Mathematics with Applications, Vol. 21, 1991, pp. 65-78. doi:10.1016/0898-1221(91)90216-Q

[14] N, Iraniparast, "A Boundary Value Problem for the Wave
Equation," International Journal of Mathematics and Mathematical Sciences, Vol. 22, No. 4, 1999, pp. 835845. doi:10.1155/S0161171299228359

[15] T. Kalmenov, "On the Spectrum of a Selfadjoint Problem for the Wave Equation," Akad. Nauk. Kazakh. SSR, Vestnik, Vol. 1, No. 3, 1983, pp. 63-66.

[16] R. A. Adams, "Sobolev Spaces," Academic Press, New York, 1975.

[17] A. Shermenew, "Nonlinear Wave Equation in Special Coordinates," Journal of Nonlinear Mathematical Physics, Vol. 11, No. 2, 2004, pp. 110-115. doi:10.2991/jnmp.2004.11.s1.14

[18] E. Burman, "Adaptive Finite Element Methods for Compressible Two-Phase Flow," Mathematical Methods in the Applied Sciences, Vol. 10, No. 2, 2000, pp. 963-989. doi:10.1016/S0218-2025(00)00049-5

[19] C. Johnson and A. Szepessy, "Adaptive Finite Element Methods for Conservation Laws Based on a Posteriori Error Estimates," Communications on Pure and Applied Mathematics, Vol. 48, No. 3, 1995, pp. 199-234. doi:10.1002/cpa.3160480302

[20] R. Sandboge, "Adaptive Finite Element Methods for Systems of Reaction-Diffusion Equations," Computer Methods in Applied Mechanics and Engineering, Vol. 166, No. 3, 1998, pp. 309-328. doi:10.1016/S0045-7825(98)00093-0

[21] P. G. Ciarlet, "The Finite Element Method for Elliptic Problems," Amesterdam, North Holland, 1987. 